\title{
Higgs mediated CLFV processes $\mu N(e N) \rightarrow \tau X$ via gluon operators
}

Masato Yamanaka*†

Maskawa Institute, Kyoto Sangyo University, Kyoto 603-8555, Japan

E-mail: masato.yamanaka@cc.kyoto-su.ac.jp

\section{Michihisa Takeuchi}

Kavli IPMU (WPI), The University of Tokyo, Kashiwa, Chiba 277-8583, Japan

E-mail: michihisa.takeuchi@ipmu.jp

\section{Yuichi Uesaka}

Department of Physics, Osaka University, Toyonaka, Osaka 560-0043, Japan

E-mail: uesaka@kern.phys.sci.osaka-u.ac.jp

We revisit charged lepton flavor violating (CLFV) scattering processes $\ell_{i} N \rightarrow \tau X\left(\ell_{i} \ni e, \mu\right)$ mediated by Higgs. We point out that a new subprocess $\ell_{i} g \rightarrow \tau g$ via the effective interactions of Higgs and gluon gives the dominant contribution to $\ell_{i} N \rightarrow \tau X$ for an incident beam energy of $E_{\ell} \lesssim 1 \mathrm{TeV}$ in fixed target experiments. Furthermore, in the light of quark number conservation, we consider quark pair-production processes $\ell_{i} g \rightarrow \tau q \bar{q}$ ( $q$ denotes quarks) instead of $\ell_{i} q \rightarrow \tau q$. This corrects the threshold energy of each subprocess contributing to $\sigma\left(\ell_{i} N \rightarrow \tau X\right)$. Reevaluation of $\sigma\left(\ell_{i} N \rightarrow \tau X\right)$ including all of relevant subprocesses shows that the search for $\ell_{i} N \rightarrow \tau X$ could serve a complementary opportunity with other relevant processes to shed light on the Higgs CLFV. This article is based on Ref. [1].

The 19th International Workshop on Neutrinos from Accelerators-NUFACT2017

25-30 September, 2017

Uppsala University, Uppsala, Sweden

* Speaker.

${ }^{\dagger}$ A footnote may follow. 


\section{Introduction}

One of the most puzzling issues in particle physics is the flavor sector. Many types of solutions for this puzzle have been proposed in UV completions of the standard model (SM), and in general predict a misaligned Yukawa couplings in the mass basis, which give rise to flavor violating interactions of Higgs and fermions. The Higgs therefore could be a promising probe to the puzzle.

Charged lepton flavor violation (CLFV) is an important process to search for the Higgs flavor violation [2, 3]. We focus on the tau CLFV as they are less constrained and a sizable effect could be expected. Once one of the tau CLFV processes is discovered, another tau CLFV process and their correlation should be studied. Among the complementary reactions the tau production $\ell_{i} N \rightarrow \tau X$ is relatively less attention paid to. Here $N$ is a nucleus. This process is expected to happen in fixed target experiments at a sizable rate $\propto \rho$, where $\rho$ being target density [4] 5]. In the light of these experiments, $\ell_{i} N \rightarrow \tau X$ would be a promising probe for the Higgs CLFV.

We point out that the gluon initiated subprocess, having not considered in the literatures,

$$
\ell_{i} g \rightarrow \tau g
$$

provides a dominant contribution to $\ell_{i} N \rightarrow \tau X$ in fixed target experiments for incident beam energy of $E_{l} \lesssim 1 \mathrm{TeV}$. Furthermore, we stress the importance of quark number conservation. Since sea quarks are generated through gluon splitting, we have to include the final state with a quark pair,

$$
\ell_{i} g \rightarrow \tau q \bar{q},
$$

instead of sea quark single-production $\ell_{i} q \rightarrow \tau q$. Here $q=s, c, b, t$. The related subprocess $\ell_{i} q \rightarrow \tau q$ has been studied in Ref. [6], where they consider only the effective 4-Fermi operators involving the sea quarks and evaluate the cross sections assuming massless partons. The difference of the phase space between $\ell_{i} g \rightarrow \tau q \bar{q}$ and $\ell_{i} q \rightarrow \tau q$ is not negligible at the fixed target experiments. For example, $\tau b \bar{b}$ is kinematically allowed only when the beam energy exceeds $E_{\ell} \gtrsim 55 \mathrm{GeV}$, while it would be $E_{\ell} \gtrsim 19 \mathrm{GeV}$ when the $\tau b$ threshold is considered. We reformulate the Higgs mediated tau production by taking the new effects: (i) the effective interaction of Higgs and gluons, (ii) the quark number conservation, into account. The cross section of $\ell_{i} N \rightarrow \tau X$ drastically changes from the previous estimation. It largely affects the search for this process in next generation experiments.

\section{CLFV scattering $\ell_{i} N \rightarrow \tau X$ with gluon operators}

The Lagrangian for the Higgs CLFV scattering $\ell_{i} N \rightarrow \ell_{j} X$ is $\mathscr{L}=\mathscr{L}_{\mathrm{SM}}+\mathscr{L}_{\mathrm{CLFV}}$, where

$$
\mathscr{L}_{\mathrm{SM}}=-\sum_{q} y_{q} h \bar{q} q+g_{h g g} h G_{\mu \nu}^{a} G^{a \mu \nu}, \quad \mathscr{L}_{\mathrm{CLFV}}=-\rho_{i j} \bar{\ell}_{j} P_{L} \ell_{i} h-\rho_{j i} \bar{\ell}_{j} P_{R} \ell_{i} h .
$$

Here $G_{\mu \nu}^{a}$ is gluon field strength, and $g_{\text {hgg }}$ is an effective coupling. CLFV interactions are parametrized by the couplings $\rho_{i j}$, where $i$ and $j$ are flavor indices, and $i \neq j$.

The effective coupling $g_{\text {hgg }}$ is generated by the diagrams where quarks are running (Fig.11(a)), and is derived as a function of the momentum transfer of the Higgs $q_{h}\left(q_{h}^{2}=-Q^{2}<0\right)$ as [8, 9 ,

$$
g_{h g g}=\sum_{q=c, b, t} \frac{\alpha_{s}}{8 \pi v} \frac{4 m_{q}^{2}}{q_{h}^{2}}\left[1+\left(1-\frac{4 m_{q}^{2}}{q_{h}^{2}}\right) f\left(\frac{4 m_{q}^{2}}{q_{h}^{2}}\right)\right], \quad f(r)=-\frac{1}{4} \log ^{2}\left[-\frac{1+\sqrt{1-r}}{1-\sqrt{1-r}}\right] \quad(r<0) .
$$




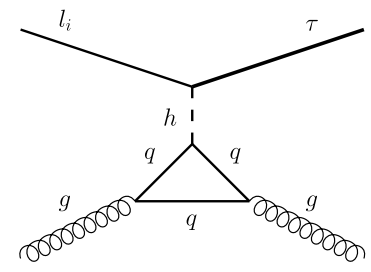

(a)

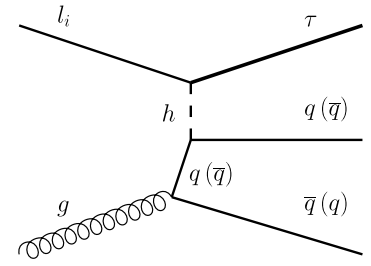

(b)

Figure 1: Subprocesses of $\ell_{i} N \rightarrow \tau X$. (a) $\ell_{i} g \rightarrow \tau g$ via gluon operator. $(b) \ell_{i} g \rightarrow \tau q \bar{q}$.

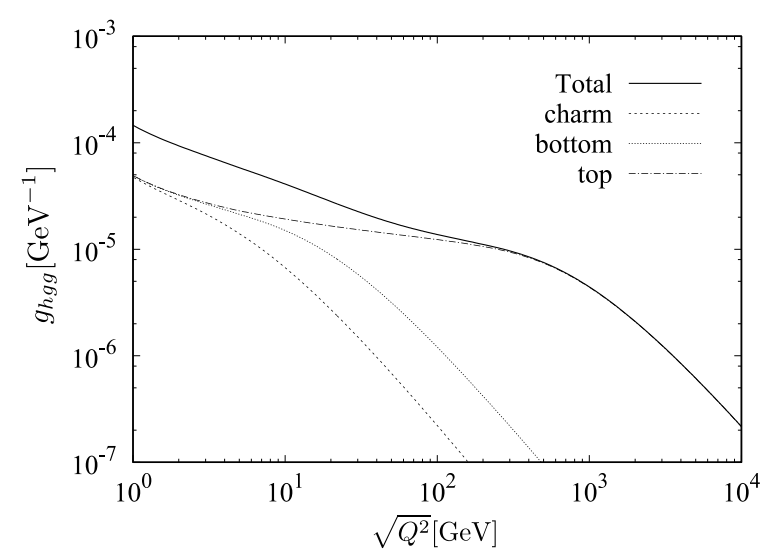

Figure 2: Scale dependence of $g_{h g g}$ and each quark contribution.

where $v=246 \mathrm{GeV}$ is the vacuum expectation value of the Higgs field, $\alpha_{s}=g_{s}^{2} / 4 \pi$, and $m_{q}$ is the mass of the running quark. Note that the function $f(r)$ has no imaginary part since $q_{h}^{2}<0$ for the scattering $\ell_{i} g \rightarrow \tau g$. This is different from the Higgs production at the LHC, where the scale is fixed at $q_{h}^{2}=m_{h}^{2}$. We count only charm, bottom, and top contributions for the coupling. Each contribution approaches to a constant for smaller $Q^{2}$ since the loop function has the asymptotic form as $r[1+(1-r) f(r)] \rightarrow 2 / 3$ (For $r \rightarrow-\infty, Q^{2} \rightarrow 0$ ). Therefore, bottom and charm quark also contribute. This is different from the case at LHC, where the contribution is only via the top loop as $q_{h}^{2}=m_{h}^{2}$. Fig. 2 2 shows the $Q^{2}$ dependence of $g_{h g g}$ and each contribution. Due to the constructive contributions there exists an enhancement relative to the case with top contribution only.

The Lagrangian (2.1) describes the two types of subprocesses, $\ell_{i} g \rightarrow \tau g$ (Fig.11(a)) and $\ell_{i} g \rightarrow$ $\tau q \bar{q}$ (Fig. 1(b)). The total cross section is formulated as

$$
\sigma_{\ell_{i} N \rightarrow \tau X}=\sum_{\hat{X}=g, q \bar{q}} \int d x d y \int_{0}^{1} d \xi \frac{d^{2} \hat{\sigma}_{\ell_{i} g \rightarrow \tau \hat{X}}}{d x d y} f_{g}\left(\xi, Q^{2}\right),
$$

where $x \equiv Q^{2} / 2 P \cdot q_{h}$ is the Bjorken variable and $y \equiv 2 P \cdot q_{h} / 2 P \cdot p_{i}$ is the measure of inelasticity. Here, $P$ and $p_{i}$ denote momenta of the initial nucleon and the initial lepton. Note that the momentum transfer $q_{h}=p_{i}-p_{\tau}$ is defined only with the initial lepton and the final tau momenta but not with the momentum related with $\hat{X}$. The ranges of $x$ and $y$ are given in Refs. [11,10, 11. The gluon parton distribution function (PDF) is denoted as $f_{g}\left(\xi, Q^{2}\right)$ and $\xi$ is the four-momentum fraction of the nucleon carried by the parton, $p_{g}=\xi P$. The range of $\xi$ depends on the subprocess. 
The parton level differential cross section of $\ell_{i} g \rightarrow \tau g$ in a massless limit of incident lepton is

$$
\frac{d \hat{\sigma}_{\ell_{i} g \rightarrow \tau g}}{d x d y}=\frac{Q^{4}\left(Q^{2}+m_{\tau}^{2}\right)}{8 \pi \hat{s}} \frac{\left|g_{\text {hgg }}\right|^{2}\left(\left|\rho_{i \tau}\right|^{2}+\left|\rho_{\tau i}\right|^{2}\right)}{\left(Q^{2}+m_{h}^{2}\right)^{2}} \delta(\xi-x) .
$$

The invariant mass of the system is $\hat{s}=\left(p_{i}+p_{g}\right)^{2}$. We have the relation $\xi=x$ as the outgoing gluon is massless. In the same limit, the parton level differential cross section of $\ell_{i} g \rightarrow \tau q \bar{q}$ is given by

$$
\begin{aligned}
& \frac{d \hat{\sigma}_{\ell_{i g} \rightarrow \tau q \bar{q}}}{d x d y}=\frac{\alpha_{s} y\left(Q^{2}+m_{\tau}^{2}\right)}{64 \pi^{2} \xi\left(Q^{2}+w^{2}\right)^{2}}\left\{2 K w^{2}\left(4 m_{q}^{2}+Q^{2}\right)+\left[\left(Q^{2}+w^{2}\right)^{2}\right.\right. \\
& \left.\left.-2\left(4 m_{q}^{2}+Q^{2}\right)\left(w^{2}-2 m_{q}^{2}\right)\right] \log \left|\frac{1+K}{1-K}\right|\right\} \frac{y_{q}^{2}\left(\left|\rho_{i \tau}\right|^{2}+\left|\rho_{\tau i}\right|^{2}\right)}{\left(Q^{2}+m_{h}^{2}\right)^{2}} \theta\left(\xi-x \frac{Q^{2}+4 m_{q}^{2}}{Q^{2}}\right),
\end{aligned}
$$

where $K \equiv \sqrt{1-4 m_{q}^{2} / w^{2}}$, and $w^{2}=\left(p_{g}+q_{h}\right)^{2}=\left(p_{q}+p_{\bar{q}}\right)^{2}=Q^{2}(\xi / x-1)$ is the invariant mass of the final quark and anti-quark system. For $\ell_{i} g \rightarrow \tau q \bar{q}$, to correct the finite mass effect of the outgoing quarks $m_{q}, \xi=x\left(Q^{2}+w^{2}\right) / Q^{2}$ is taken [12].

We briefly discuss $\ell_{i} N \rightarrow \tau X$ mediated by other heavy particles which (in)directly couples with the gluon, e.g., heavy Higgses $H$ and $A$ in two Higgs doublet models. As long as those particles are heavy enough we can describe $\ell_{i} g \rightarrow \tau g$ using the following effective operators,

$$
\mathscr{L}_{\text {eff }}=\overline{\ell_{j}}\left(C_{i j} P_{L}+C_{j i} P_{R}\right) \ell_{i} G_{\mu v}^{a} G^{a \mu v}
$$

Constraints are $\left|C_{\mu \tau}\right|^{2}+\left|C_{\tau \mu}\right|^{2}<7.4 \times 10^{-18} \mathrm{GeV}^{-6}$ and $\left|C_{e \tau}\right|^{2}+\left|C_{\tau e}\right|^{2}<8.1 \times 10^{-18} \mathrm{GeV}^{-6}$ [14], which coming from $\operatorname{BR}\left(\tau \rightarrow \mu \pi^{+} \pi^{-}\right)<2.1 \times 10^{-8}$ and $\operatorname{BR}\left(\tau \rightarrow e \pi^{+} \pi^{-}\right)<2.3 \times 10^{-8}$ [13]. Note that these constraints are much weaker than those assuming only via the SM Higgs. The differential cross section of $\ell_{i} g \rightarrow \tau g$ is

$$
\frac{d \hat{\sigma}_{\ell_{i} g \rightarrow \ell_{j} g}^{\text {contac }}}{d x d y}=\frac{Q^{4}\left(Q^{2}+m_{j}^{2}\right)}{8 \pi \hat{s}}\left(\left|C_{i j}\right|^{2}+\left|C_{j i}\right|^{2}\right) \delta(\xi-x) .
$$

\section{Numerical analysis}

We perform a numerical analysis. We restrict the phase space integration with $W^{2}>(1.5 \mathrm{GeV})^{2}$ and $Q^{2}>(1 \mathrm{GeV})^{2}$ to ensure that the parton model picture is valid, where $W^{2}=\left(P+q_{h}\right)^{2}$ is the hadronic invariant mass. We consider only the DIS regime and ignore other resonant effects which are known to be sub-dominant [10].

First we consider fixed target experiments. Fig. 3 shows the cross section of $\ell_{i} N \rightarrow \tau X$ as a function of lepton beam energy. Due to the large gluon PDF and no phase space suppression, the new subprocess $\ell_{i} g \rightarrow \tau g$ leads to large enhancement. The ratio between $\sigma\left(\ell_{i} N \rightarrow \tau X\right)$ with and without the new subprocess is $7.8(1.8)$ for $E_{\ell}=50 \mathrm{GeV}(500 \mathrm{GeV})$. The subprocess $\ell_{i} g \rightarrow \tau c \bar{c}$ $\left(\ell_{i} g \rightarrow \tau b \bar{b}\right)$ only starts to be relevant at $E_{\ell} \sim 100 \mathrm{GeV}(500 \mathrm{GeV})$. Inclusion of the $g_{\text {hgg }}$ coupling enhancement shown in Fig. 2 is also important. Typically, it provides a factor of $3 \sim 7$ enhancement 


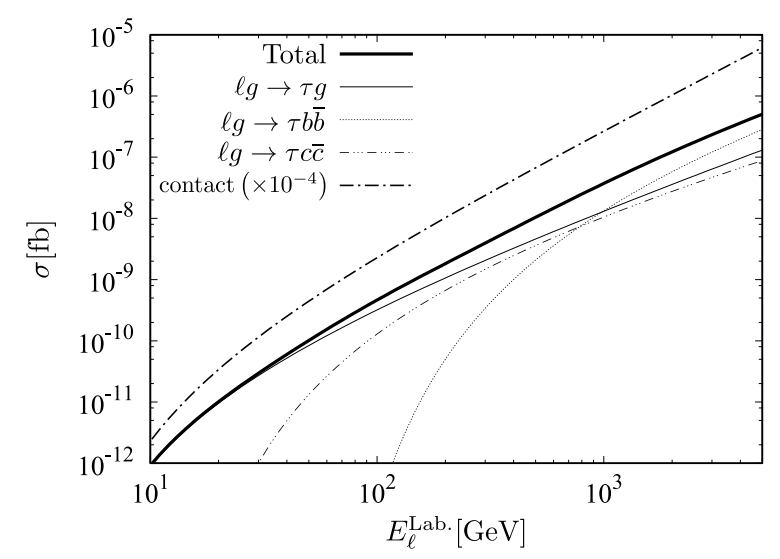

Figure 3: Cross sections of $\ell_{i} N \rightarrow \tau X$ as a function of lepton beam energy. The dot-dashed line shows the cross section for the effective interaction (2.6) for $\left|C_{\ell \tau}\right|^{2}+\left|C_{\tau \ell}\right|^{2}=8.10 \times 10^{-18} \mathrm{GeV}^{-6}$ and other lines show total and partial cross sections mediated by the Higgs for $\sqrt{\left|\rho_{\ell \tau}\right|^{2}+\left|\rho_{\tau \ell}\right|^{2}}=2.4 \times 10^{-3}$.

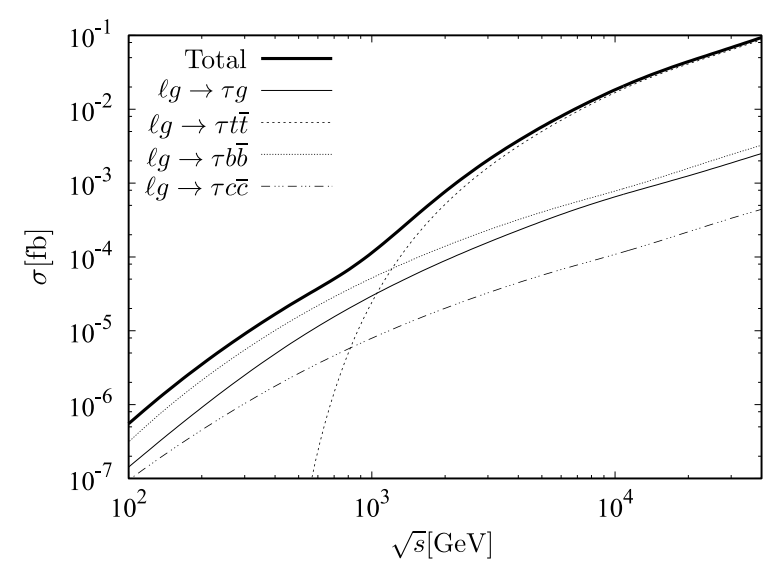

Figure 4: Total and partial cross sections of Higgs mediated scattering $\ell_{i} N \rightarrow \tau X$ for beam collision experiments. We take maximally allowed CLFV Yukawa couplings.

relative to the case with top contribution only. Event rate of $\ell_{i} N \rightarrow \tau X$ process at the fixed target experiments is estimated as [15],

$$
N \simeq 6 \times 10^{-16} \cdot N_{\ell_{i}}\left(\frac{\sigma_{\ell_{i} N \rightarrow \tau X}}{1 \mathrm{fb}}\right)\left(\frac{T_{m}}{1 \mathrm{gcm}^{-2}}\right),
$$

where $N_{\ell_{i}}$ is the intensity of $\ell_{i}$ per year, and $T_{m}$ is the target mass in unit of $\mathrm{g} \mathrm{cm}^{-2} . \mathscr{O}(10)\left(\mathscr{O}\left(10^{3}\right)\right)$ events of $\ell_{i} N \rightarrow \tau X$ are expected per year for the electron beam energy of an upgrade option in ILC (PWFA), $E_{e}=500 \mathrm{GeV}(5 \mathrm{TeV}), T_{m}=100 \mathrm{~g} \mathrm{~cm}^{-2}$, and the electron intensity $N_{e}=10^{22} /$ year.

The scattering cross section assuming the contact operator with the maximally allowed value is shown in a dot-dashed line. For muon options, we require the neutrino factories, which would reach at $N_{\mu}=10^{20} /$ year with a beam energy of $\mathscr{O}(100) \mathrm{GeV}$ to provide $\mathscr{O}(10)$ events/year. The currently available intensity is not enough, for example, $N_{\mu}=10^{15} /$ year in COMPASS II experiment [16] and $N_{\mu}=10^{19} /$ year with a lower beam energy in COMET [17] in future.

Next, we turn to the collider experiments. The cross section $\sigma(\ell N \rightarrow \tau X)$ as a function of the collision energy is shown in Fig. 4. As the collision energy $\sqrt{s}$ increases the cross section 
grows rapidly. When it reaches at $\sim 2 m_{t}$, a subprocess $\ell_{i} g \rightarrow \tau t \bar{t}$ starts to contribute and becomes dominant for $\sqrt{s} \gtrsim 1 \mathrm{TeV}$. A future electron-proton beam collision experiment TLHeC (VHE-TLHeC) plans to achieve a $\sqrt{s} \simeq 1.3(3.5) \mathrm{TeV}$ with a luminosity of $\sim 10^{35} \mathrm{~cm}^{-2} \mathrm{~s}^{-1}$, i.e., $\mathscr{O}(1000) \mathrm{fb}^{-1} /$ year. We expect $\mathscr{O}(100)$ events for the maximal allowed CLFV coupling.

\section{Summary}

The Higgs mediated CLFV scattering has been reconsidered by taking (i) new subprocess $\ell_{i} g \rightarrow \tau g$, and (ii) quark number conserving subprocess $\ell_{i} g \rightarrow \tau q \bar{q}(q=c, b, t)$, into account. At fixed target experiments with $E_{\ell} \lesssim 1 \mathrm{TeV}$, the cross section $\sigma\left(\ell_{i} N \rightarrow \tau X\right)$ is enhanced more than about twice by the subprocess $\ell_{i} g \rightarrow \tau g$. The associated quarks are only produced in pairs in $\ell_{i} N \rightarrow$ $\tau X$, and hence $\sigma\left(\ell_{i} N \rightarrow \tau q \bar{q}\right)$ starts to be relevant on higher beam energy than that estimated in the previous works wherein the phase space suppression is not considered. $\mathscr{O}(10)-\mathscr{O}\left(10^{3}\right)$ events of $\ell_{i} N \rightarrow \tau X$ could be expected in both fixed target experiments and beam collision experiments. Our results hold for other CLFV mediators which couple with gluon and/or heavy quarks.

\section{Acknowledgments}

This work is supported in part by the JSPS Grant-in-Aid for Scientific Research Numbers JP16H03991, JP16H02176, 17H05399 (M.T.), 16K05325 and 16K17693 (M.Y.). MT is supported by World Premier International Research Center Initiative (WPI Initiative), MEXT, Japan.

\section{References}

[1] M. Takeuchi, Y. Uesaka and M. Yamanaka, Phys. Lett. B 772 (2017) 279

[2] A. Goudelis, O. Lebedev and J. h. Park, Phys. Lett. B 707 (2012) 369.

[3] R. Harnik, J. Kopp and J. Zupan, JHEP 1303 (2013) 026.

[4] S. N. Gninenko, M. M. Kirsanov, N. V. Krasnikov and V. A. Matveev, Mod. Phys. Lett. A 17 (2002) 1407.

[5] M. Sher and I. Turan, Phys. Rev. D 69 (2004) 017302.

[6] S. Kanemura, Y. Kuno, M. Kuze and T. Ota, Phys. Lett. B 607 (2005) 165.

[7] V. Khachatryan et al. [CMS Collaboration], Phys. Lett. B 763 (2016) 472.

[8] H. M. Georgi, S. L. Glashow, M. E. Machacek and D. V. Nanopoulos, Phys. Rev. Lett. 40 (1978) 692.

[9] M. Spira, A. Djouadi, D. Graudenz and P. M. Zerwas, Nucl. Phys. B 453 (1995) 17.

[10] K. Hagiwara, K. Mawatari and H. Yokoya, Nucl. Phys. B 668 (2003) 364.

[11] C. H. Albright and C. Jarlskog, Nucl. Phys. B 84 (1975) 467.

[12] H. Georgi and H. D. Politzer, Phys. Rev. Lett. 36 (1976) 1281.

[13] Y. Miyazaki et al. (Belle Collaboration), Phys. Lett. B 719 (2013) 346.

[14] A. Celis, V. Cirigliano and E. Passemar, Phys. Rev. D 89 (2014) no.9, 095014.

[15] A. Abada, V. De Romeri, J. Orloff and A. M. Teixeira, arXiv:1612.05548 [hep-ph].

[16] F. Gautheron et al. [COMPASS Collaboration], SPSC-P-340, CERN-SPSC-2010-014.

[17] Y. G. Cui et al. [COMET Collaboration], KEK-2009-10. 